\title{
Noninferiority of Preservative-free Versus BAK-preserved Latanoprost-timolol Fixed Combination Eye Drops in Patients With Open-angle Glaucoma or Ocular Hypertension
}

\author{
Florent Aptel, MD, ${ }^{*}$ Norbert Pfeiffer, MD, $\dagger$ Stefanie Schmickler, MD, $\neq$ \\ Jonathan Clarke, MD, \& Cosme Lavin-Dapena, MD,\| \\ Javier Moreno-Montañés, MD, Tomasz Żarnowski, MD,\# \\ Adrienne Csutak, MD, ** Tiia Jugaste, MD, †† Lāsma Volksone, MD, $+\neq$ \\ Yury S. Astakhov, MD, $\S \S$ Laurent Coupier, MD, \|\| \\ Jean-Philippe Nordmann, MD, $1 \uparrow$ Ingeborg Stalmans, MD, PhD,\#\# \\ and the T2347 Study Group
}

Précis: Noninferiority of efficacy was demonstrated for a preser-
vative-free latanoprost-timolol fixed combination compared with a
BAK-containing formulation at 84 days after treatment in patients BAK-containing formulation at 84 days after treatm
with open-angle glaucoma or ocular hypertension.

Purpose: The purpose of this study was to compare the effect on intraocular pressure and safety of preservative-free latanoprosttimolol fixed combination (T2347) to benzalkonium chloride-preserved latanoprost-timolol fixed combination in patients with open-angle glaucoma or ocular hypertension.

Methods: Phase III, randomized, parallel-group, investigatormasked study in 10 countries. A total of 242 patients aged 18 years or older with open-angle glaucoma or ocular hypertension in both eyes controlled with a preserved latanoprost-timolol fixed combination $(15.7 \pm 2.4 \mathrm{~mm} \mathrm{Hg}$ overall before inclusion) were randomized at day 0 with no washout period to receive the preservativefree alternative T2347 $(\mathrm{N}=127)$ or remain on the preserved comparator $(\mathrm{N}=115)$ for 84 days. Intraocular pressure changes from day 0 were measured at 9:00 am ( \pm 1 hour) on day 42 and day 84 , and noninferiority of $\mathrm{T} 2347$ to the preserved comparator was analyzed statistically at day 84 . Safety parameters were also reported.

Results: The mean change in intraocular pressure from baseline to day 84 was $-0.49 \pm 1.80 \mathrm{~mm} \mathrm{Hg}$ for preservative-free T2347 and $-0.49 \pm 2.25 \mathrm{~mm} \mathrm{Hg}$ for the preserved comparator. These results met the noninferiority limits. Similar results were observed at day 42. There was no difference between groups in the incidence of adverse events or ocular signs. The total ocular symptoms score was better for T2347 than BPLT upon instillation at day $84(45.9 \%$ / $44.3 \% / 9.8 \%$ of patients with improvement/no change/worsening vs. $33.6 \% / 47.3 \% / 19.1 \% ; P=0.021$, reflecting improvements in individual symptoms such as irritation/burning/stinging $(P<0.001)$, and itching $(P<0.01)$ on day 84 .

Conclusions: Preservative-free latanoprost-timolol fixed combination T2347 showed noninferior efficacy compared with the preserved comparator and was well tolerated.

Key Words: glaucoma, ocular hypertension, preservative-free, latanoprost-timolol

( J Glaucoma 2019;28:498-506)

Received for publication November 19, 2018; accepted March 2, 2019.

From the *CHU de Grenoble/University Hospital of Grenoble, Université Grenoble Alpes, Grenoble; \|\| Centre Hospitalier du Pays d'Aix, Service Ophtalmologie, Aix-en-Provence; 9 - Ophthalmology, CHNO des Quinze-Vingts, Paris, France; †Department of Ophthalmology, Mainz University Medical Center, Mainz; †Augen-Zentrum-Nordwest, Augenpraxis Ahaus, Ahaus, Germany; §NIHR Biomedical Research Centre for Ophthalmology, Moorfields Eye Hospital and UCL Institute of Ophthalmology, London, UK; \#Hospital Universitario La Paz, Madrid; ๆClínica Universidad de Navarra, Pamplona, Spain; \#Department of Diagnostics and Microsurgery of Glaucoma, Medical University, Lublin, Poland; **Department of Ophthalmology, Faculty of Medicine, University of Debrecen, Debrecen, Hungary; ††Clinic of Ophthalmology, Tallinn, Estonia; + ț Volksone's Practice in Ophthalmology, Lavolks Ltd, Riga, Latvia; §§Department of Ophthalmology, Academician I.P. Pavlov First St. Petersburg State Medical University, St. Petersburg, Russia; and \#\#Department of Ophthalmology, University Hospitals UZ Leuven, Leuven, Belgium.

Presented at the Annual Meeting of the Association for Research in Vision and Ophthalmology (ARVO), Baltimore, MD, May 7-11, 2017, and at the World Glaucoma Congress, Helsinki, Finland, June 28-July 1, 2017.

F.A.: study design, literature search, data analysis, and evidence synthesis, and drafting and revising of the manuscript. I.S.: study design, data collection and screening, data analysis and evidence synthesis, and drafting and revising the manuscript. N.P., S.S., J.C., C.L.-D., J.M.-M., T.Ż., A.C., T.J., L.V., Y.S.A., L.C., and J.-P.N.: study design, data collection, screening, and revising of the manuscript.

Sponsored by Laboratoires Théa, Clermont-Ferrand, France, which also provided funding for medical writing assistance for this manuscript. The sponsor participated in study design, study conduct, data collection, data management, data analysis, interpretation of the data, and preparation of the manuscript.

Disclosure: F.A. is a consultant for, and has received nonfinancial support from, Laboratoires Théa. S.S. and J.C. are consultants for, or have received a research grant and nonfinancial support from Laboratoires Théa and were investigators in this study. J.P.N. is a consultant for, or has received a research grant and nonfinancial from Laboratoires Théa and Allergan, and was an investigator in this study. I.S. is a consultant for, or has received a research grant and nonfinancial support from Laboratoires Théa, Allergan, Eyetech Care, Alcon, and Santen, and was an investigator in this study. N.P., C.L.-D., J.M.-M., T.Ż., A.C., T.J., L.V., Y.S.A., L.C., have received a research grant and nonfinancial support from Laboratoires Théa and were investigators in this study.

Reprints: Ingeborg Stalmans, MD, PhD, University Hospitals UZ Leuven, Department of Ophthalmology Herestraat 49, 3000 Leuven, Belgium (e-mail: ingeborg.stalmans@uzleuven.be).

Supplemental Digital Content is available for this article. Direct URL citations appear in the printed text and are provided in the HTML and PDF versions of this article on the journal's website, www.glaucomajournal.com.

Copyright (C) 2019 The Author(s). Published by Wolters Kluwer Health, Inc. This is an open-access article distributed under the terms of the Creative Commons Attribution-Non Commercial-No Derivatives License 4.0 (CCBY-NC-ND), where it is permissible to download and share the work provided it is properly cited. The work cannot be changed in any way or used commercially without permission from the journal.

DOI: $10.1097 / \mathrm{IJG} .0000000000001248$ 
T opical eye drop formulations containing prostaglandin analogues or $\beta$-blockers are routinely used as a first-line treatment to prevent glaucoma progression by reducing intraocular pressure (IOP). ${ }^{1}$ However, in $40 \%$ to $75 \%$ of open-angle glaucoma (OAG) patients, monotherapy fails to achieve a satisfactory reduction in IOP after $>2$ years of treatment, and combined therapy is needed. ${ }^{2}$ The administration of separate component medications could be associated with reduced patient compliance to the treatment schedule as the number of instillations increases. ${ }^{3-6}$ Moreover, subsequent treatments given in this way can lead to washout of the previous one. Both of these factors lead to reduced efficacy of unfixed combined treatments. ${ }^{7,8}$

Fixed combination formulations offer improved convenience, adherence to the treatment regimen, and effectiveness as well as cost reductions. ${ }^{9-13}$ Clinical studies with a fixed combination of latanoprost $0.005 \%$ and timolol $0.5 \%$ have demonstrated its suitability in patients with OAG or ocular hypertension (OHT) with better efficacy than either component administered alone. ${ }^{14-17}$ Because of these advantages, the use of fixed combinations has been recommended by the European Glaucoma Society ${ }^{1}$ and is preferred over unfixed combinations in many countries.

Clinical studies with formulations containing benzalkonium chloride (BAK), an antimicrobial preservative, ${ }^{18,19}$ have shown a higher incidence of ocular symptoms compared with preservative-free formulations and a clear association of the preservative with the occurrence and severity of ocular problems such as corneal toxicity and conjunctival damage. ${ }^{20-22}$ An alternative preservative to BAK including boric acid and zinc chloride has been used in a latanoprost-timolol fixed combination formulation with similar efficacy and no difference in the incidence of treatment-emergent adverse events. ${ }^{23}$ However, the development of preservative-free combination formulations is considered to be an important goal, and the European Medicines Agency issued a public statement on ophthalmic formulations that "for long-term treatment, formulations without preservatives are considered to be valuable." 24 Preservative-free latanoprost-timolol fixed combination formulations, such as T2347, are expected to be better tolerated than BAK-preserved latanoprost-timolol formulations over the long term. This is particularly relevant for glaucoma patients, who have a higher prevalence of ocular surface disease than the general population, ${ }^{1}$ and for whom improved tolerability can be associated with better compliance and therefore improved long-term effectiveness., ${ }^{9,20}$ Better tolerability of preservative-free formulations has a positive impact on patient quality of life. In addition, the stability at room temperature of eye drops such as preservative-free T2347 removes the need for cold-chain storage, which may further improve treatment convenience and compliance..$^{23,25}$

As glaucoma requires daily, long-term IOP-lowering treatment and preserved formulations are associated with tolerability issues, this study was performed to establish the noninferiority of the efficacy of T2347 compared with a BAK-preserved latanoprost-timolol (BPLT) fixed combination after 84 days of treatment in patients who had previously been receiving the preserved formulation, and also to compare their safety and tolerability profiles.

\section{MATERIALS AND METHODS}

\section{Study Design and Patients}

This was a phase III, international, randomized, parallelgroup, investigator-masked study in 47 centers in 10 countries -
Belgium (3 centers), Estonia (3), France (5), Germany (5), Hungary (4), Latvia (1), Poland (7), Russia (3), Spain (11), and the United Kingdom (5). The study was conducted in compliance with Good Clinical Practice (ICH-E6), the ethical principles of the Declaration of Helsinki (2004), and applicable local legislation. Before enrollment, written informed consent was obtained from each patient. The study took place between December 2014 and November 2015 (ClinicalTrials.gov identifier: NCT02278614).

Patients aged 18 years or older with OAG or OHT in both eyes that were already treated and well-controlled for at least 2 months before study entry with latanoprost $0.005 \%$ and timolol $0.5 \%$ fixed combination preserved eye drops (any brand) were eligible for inclusion if the following criteria were fulfilled: IOP $\leq 18 \mathrm{~mm} \mathrm{Hg}$ in both eyes at the inclusion visit, a history of IOP insufficiently controlled with first-line monotherapy and a history of additional IOP reduction following a switch to latanoprost $0.005 \%$ and timolol $0.5 \%$ fixed combination preserved eye drops (Table 1), and corneal thickness of 500 to $600 \mu \mathrm{m}$ in both eyes.

The main ophthalmic exclusion criteria included significant worsening between the last 2 visual field assessments ( $\geq 6$ mo between assessments); severe stage of glaucoma; best-corrected far visual acuity $\leq 1 / 10$ in at least one eye; ocular infection, trauma, or inflammation in the previous 3 months; presence of at least one severe objective sign [McMonnies maximum (grade 5) conjunctival hyperemia or maximum (grade 3) blepharitis]; diagnosed severe dry eye; corneal ulceration; palpebral abnormalities; and any other abnormality that could prevent accurate study assessments. Other exclusion criteria included history of corneal refractive surgery; intraocular laser procedures ( 3 mo before or during the study); other ocular surgery ( 6 mo before and during the study); any topical ocular treatment (except preservative-free artificial tears or preservative-free antiallergic eye drops); and use of contact lenses (for $1 \mathrm{wk}$ before or during the study).

At day 0 (D0, inclusion), patients were randomized to either receive a preservative-free fixed combination of latanoprost $0.005 \%$ and timolol $0.5 \%$ [T2347 (Fixaprost), presented in single-dose units; Laboratoires Théa, ClermontFerrand, France] or to continue with a BAK-preserved formulation [BPLT (Xalacom), containing $0.2 \mathrm{mg} / \mathrm{mL}$ BAK, presented in multidose bottles; Pfizer, New York, NY] without a washout period. The randomized code was generated by Lincoln (Boulogne-Billancourt, France), using random permuted blocks with a fixed block size of 4 , in a 1:1 ratio. All eligible subjects were assigned the sequential randomization number available at the site. To ensure investigator-masking, personnel other than the investigator dispensed all study treatments. Patients were to instill one drop in each eye at 9 pm $( \pm 1 \mathrm{~h})$ daily for 84 days. Follow-up visits took place at the study site at D42 \pm 3 days (D42) and D84 \pm 7 days (D84).

\section{Efficacy Assessments}

\section{Primary Efficacy Variable}

The primary efficacy variable was the change in mean IOP from D0 to D84 measured using a calibrated Goldmann applanation tonometer at $9 \mathrm{am}( \pm 1 \mathrm{~h})$ in the worse eye (ie, the eye with the highest IOP or the right eye if IOP was the same in both eyes). Two measurements were taken; if these differed by $>2 \mathrm{~mm} \mathrm{Hg}$, a third reading was taken. The average value was used in the analysis. 
TABLE 1. Demographic and Baseline Assessments for the Worse Eye (ITT/Safety set)

\begin{tabular}{|c|c|c|c|}
\hline & $\begin{array}{c}\mathrm{T} 2347 \\
(\mathrm{~N}=127) \\
\end{array}$ & $\begin{array}{c}\text { BPLT } \\
(\mathrm{N}=115)\end{array}$ & $\begin{array}{c}\text { Overall } \\
(\mathrm{N}=\mathbf{2 4 2})\end{array}$ \\
\hline \multicolumn{4}{|l|}{ Sex, n (\%) } \\
\hline Male & $47(37.0)$ & $46(40.0)$ & $93(38.4)$ \\
\hline Female & $80(63.0)$ & $69(60.0)$ & $149(61.6)$ \\
\hline \multicolumn{4}{|l|}{ Age (y) } \\
\hline Mean \pm SD & $65.8 \pm 10.8$ & $67.2 \pm 10.6$ & $66.4 \pm 10.7$ \\
\hline Range & $27-87$ & $35-91$ & $27-91$ \\
\hline \multicolumn{4}{|l|}{ Diagnosis, n (\%) } \\
\hline OAG & $95(74.8)$ & $99(86.1)$ & $194(80.2)$ \\
\hline OHT & $32(25.2)$ & $16(13.9)$ & $48(19.8)$ \\
\hline \multicolumn{4}{|c|}{ Diagnosis category, n (\%) } \\
\hline $\begin{array}{l}\text { Exfoliative } \\
\text { glaucoma }\end{array}$ & $7(5.5)$ & $7(6.1)$ & $14(5.8)$ \\
\hline $\begin{array}{l}\text { Pigmentary } \\
\text { glaucoma }\end{array}$ & $1(0.8)$ & $1(0.9)$ & $2(0.8)$ \\
\hline $\begin{array}{l}\text { Primary OAG/ } \\
\text { OHT }\end{array}$ & $119(93.7)$ & $107(93.0)$ & $226(93.4)$ \\
\hline \multicolumn{4}{|c|}{ Time from diagnosis (mo) } \\
\hline Mean \pm SD & $103.2 \pm 72.3$ & $91.2 \pm 81.1$ & $97.5 \pm 76.7$ \\
\hline \multicolumn{4}{|c|}{ Corneal thickness $(\mu \mathrm{m})$} \\
\hline Mean \pm SD & $546.7 \pm 27.2$ & $549.5 \pm 26.0$ & $548 \pm 26.6$ \\
\hline \multicolumn{4}{|c|}{ Fundoscopy (C/D ratio) } \\
\hline Mean \pm SD & $0.50 \pm 0.21$ & $0.51 \pm 0.22$ & $0.50 \pm 0.21$ \\
\hline$<0.7$, n $(\%)$ & $95(74.8)$ & $81(70.4)$ & $176(72.7)$ \\
\hline$\geq 0.7, \mathrm{n}(\%)$ & $32(25.2)$ & $34(29.6)$ & $66(27.3)$ \\
\hline \multicolumn{4}{|c|}{ IOP history } \\
\hline \multicolumn{4}{|c|}{ IOP with monotherapy (before combination therapy) } \\
\hline $\mathrm{n}^{\dagger}$ & 92 & 89 & 181 \\
\hline $\begin{array}{l}\text { Mean } \pm \mathrm{SD} \\
(\mathrm{mm} \mathrm{Hg})\end{array}$ & $21.1 \pm 3.5$ & $20.5 \pm 3.4$ & $20.8 \pm 3.5$ \\
\hline$[95 \% \mathrm{CI}]$ & {$[20.3,21.8]$} & {$[19.7,21.2]$} & {$[20.3,21.3]$} \\
\hline \multicolumn{4}{|c|}{ IOP with combination therapy* (before inclusion in the study) } \\
\hline $\mathrm{n} \dagger$ & 110 & 101 & 211 \\
\hline $\begin{array}{l}\text { Mean } \pm \text { SD } \\
(\mathrm{mm} \mathrm{Hg})\end{array}$ & $15.8 \pm 2.5$ & $15.6 \pm 2.4$ & $15.7 \pm 2.4$ \\
\hline$[95 \% \mathrm{CI}]$ & {$[15.3,16.3]$} & {$[15.1,16.0]$} & {$[15.4,16.0]$} \\
\hline
\end{tabular}

*Within 6 months.

$t \mathrm{n}=$ number of patients with available data

$\mathrm{C} / \mathrm{D}$ indicates cup to disc ratio; $\mathrm{CI}$, confidence interval; IOP, intraocular pressure; N, number of patients in group; OAG, open-angle glaucoma; OHT, ocular hypertension.

\section{Secondary Efficacy Variables}

Secondary efficacy variables were IOP in the contralateral eye at D84 and in both eyes at D42. In addition, the investigator rated global efficacy on D42 and D84 (very satisfactory, satisfactory, not very satisfactory, or unsatisfactory).

\section{Safety Assessments}

\section{Adverse Events}

Ocular and systemic adverse events (AEs) and their severity (mild, moderate, or severe) were recorded at D42 and D84. The investigator determined the potential relatedness of each AE to the study treatment (none, unlikely, possible, or definite).

\section{Other Safety Assessments}

Other safety outcome measures, separate to the AE reporting, included patient-assessed prelisted ocular symptoms upon instillation and throughout the day [irritation/burning/ stinging, itching, tearing, foreign body sensation, and eye dryness sensation; each was graded using a 4-point severity score as none (0), present but not disturbing (1), disturbing (2), or very disturbing (3), and a total severity score was calculated using the sum of the scores for each symptom]; ocular signs on slit-lamp examination [conjunctival hyperemia was scored using the McMonnies scale (0 to 5) and ocular staining using the Oxford (0 to 15 ) grading scheme]; corneal thickness; fundoscopy; visual field examinations; best-corrected far visual acuity using a Snellen chart; blood pressure and heart rate; and investigator-reported and patient-reported satisfaction of local tolerance (rated as very satisfactory, satisfactory, not very satisfactory, or unsatisfactory).

\section{Statistical Analyses}

The primary objective of this study was to demonstrate noninferiority of T2347 versus BPLT in terms of the change from baseline (D0) in mean IOP on D84 in the worse eye. The noninferiority test was based on the $95 \%$ confidence interval (CI) of the difference between the 2 groups (T2347 minus BPLT) using a mixed model for repeated measures (MMRM), including treatment, visit, treatment by visit interaction, and baseline IOP by visit interaction adjusted for baseline IOP and country. Noninferiority was concluded if the upper bound of the $95 \% \mathrm{CI}$ for the difference was $<+1.5 \mathrm{~mm} \mathrm{Hg}$. Sensitivity analyses [analysis of covariance (ANCOVA)] based on the lastobservation-carried-forward method were performed to explore any possible impact of the dropout pattern on the noninferiority analysis. Treatment comparisons for the secondary efficacy endpoints and the safety endpoints were performed using ANCOVA for quantitative parameters or the CochranMantel-Haenszel (CMH) test for ordered qualitative parameters. As changes from baseline in total subjective ocular symptoms score were not normally distributed, the changes in total symptoms scores were assessed on the basis of 3 classes [improvement $(<0) /$ no change $(0) /$ worsening $(>0)$ ] and compared between treatment groups using a $\mathrm{CMH}$ test with modified ridit scores stratified by country (post hoc analyses).

A total of 194 patients ( 97 patients in each treatment group) evaluable for the efficacy analysis would provide $90 \%$ power for the noninferiority calculation, assuming no difference between the groups and a standard deviation of $3.2 \mathrm{~mm} \mathrm{Hg}$ for the primary efficacy variable. Assuming a dropout rate of $7 \%$, it was planned to enroll a total of 210 patients (105 in each group).

Efficacy analyses were performed using the modified intent-to-treat (mITT) set (all randomized patients who received at least one dose and had at least one postbaseline efficacy evaluation); the primary efficacy analysis was confirmed using the per protocol (PP) set (all mITT patients without a major protocol deviation) and ITT set (all randomized patients who received at least one dose). Safety analyses were performed using the safety set (all enrolled patients who received at least one dose).

\section{RESULTS}

\section{Patients}

Overall, 242 patients were randomized at D0 to receive T2347 $(\mathrm{N}=127)$ or remain on BPLT treatment $(\mathrm{N}=115)$ (ITT set; Fig. 1). Of them, 122 and 110 patients, respectively, completed the study. All enrolled patients had wellcontrolled IOP before inclusion in the study $(15.7 \pm 2.4 \mathrm{~mm} \mathrm{Hg}$ overall before inclusion) (Table 1) and at baseline $(15.6 \pm 2.1$ and $15.7 \pm 2.1 \mathrm{~mm} \mathrm{Hg}$, in the $\mathrm{T} 2347$ and BPLT groups, respectively) (Table 2). There were no 
relevant differences between groups for demography and baseline assessments (Table 1).

\section{Efficacy}

\section{Primary Efficacy Variable}

The mean change in IOP at D84 was $-0.49 \pm 1.80 \mathrm{~mm} \mathrm{Hg}$ for T2347 and $-0.49 \pm 2.25 \mathrm{~mm} \mathrm{Hg}$ for BPLT in the mITT set (Table 2). Noninferiority was demonstrated at D84, as the upper limit of the $95 \%$ CI for the difference between treatments (T2347 minus BPLT) was $<1.5 \mathrm{~mm} \mathrm{Hg}(0.50 \mathrm{~mm} \mathrm{Hg}$, MMRM). Noninferiority was confirmed by the PP and ITT analyses.

The sensitivity analysis using ANCOVA also supported the result of noninferiority (upper $95 \% \mathrm{CI}: 0.54 \mathrm{~mm} \mathrm{Hg}$ ).

\section{Secondary Efficacy Variables}

In the contralateral eye, the change in IOP from baseline at D84 was $0.08 \pm 2.06 \mathrm{~mm} \mathrm{Hg}$ for T2347 and $0.14 \pm 2.25 \mathrm{~mm} \mathrm{Hg}$ for BPLT, with an upper $95 \% \mathrm{CI}$ for the treatment difference of $0.43 \mathrm{~mm} \mathrm{Hg}$ (MMRM), supporting the primary analysis. The sensitivity analysis for the contralateral eye (ANCOVA) also supported the primary analysis (upper $95 \% \mathrm{CI}: 0.44 \mathrm{~mm} \mathrm{Hg}$ ).

Similar results were seen at D42 in the worse eye, with a change in IOP of $-0.65 \pm 1.86 \mathrm{~mm} \mathrm{Hg}$ for $\mathrm{T} 2347$ and $-0.49 \pm 2.24 \mathrm{~mm} \mathrm{Hg}$ for BPLT (upper $95 \% \mathrm{CI}$ : $0.31 \mathrm{~mm} \mathrm{Hg}$ ) (Table 2). Results for the contralateral eye also showed no difference between groups at D42, supporting the results for the worse eye, with a change in IOP of $0.05 \pm 2.41 \mathrm{~mm} \mathrm{Hg}$ for T2347 and $0.05 \pm 2.18 \mathrm{~mm} \mathrm{Hg}$ for BPLT (upper $95 \% \mathrm{CI}$ : $0.52 \mathrm{mmHg}$ ).

Investigators assessed the global efficacy as satisfactory or very satisfactory with no statistically significant difference between treatments at D42 $(\geq 94.6 \% ; P=0.850, \mathrm{CMH})$ and D84 ( $\geq 97.5 \% ; P=0.862)$.

\section{Safety and Tolerability}

\section{Adverse Events}

In the T2347 group, $12(9.4 \%)$ patients experienced treatment-related ocular AEs versus $8(7.0 \%)$ patients in the BPLT group $(P=0.482)$. The most common treatmentrelated ocular AEs were eye irritation (2 and 5 patients in the T2347 and BPLT groups, respectively) and conjunctival hyperemia ( 3 and 1 patients) (Table 3 ). The incidence of treatment-related systemic AEs was similar in both treatment groups $(P=0.686)$, reported by $4(3.1 \%)$ patients in the T2347 group and $2(1.7 \%)$ patients in the BPLT group (Table 3).

Most treatment-related ocular and systemic AEs were mild or moderate in severity. No patient in either group experienced an ocular SAE; one patient experienced a systemic SAE (cerebral artery occlusion in the BPLT group), but this was not considered to be treatment related, and the patient recovered without sequelae. Three patients were withdrawn because of treatment-related ocular AEs of

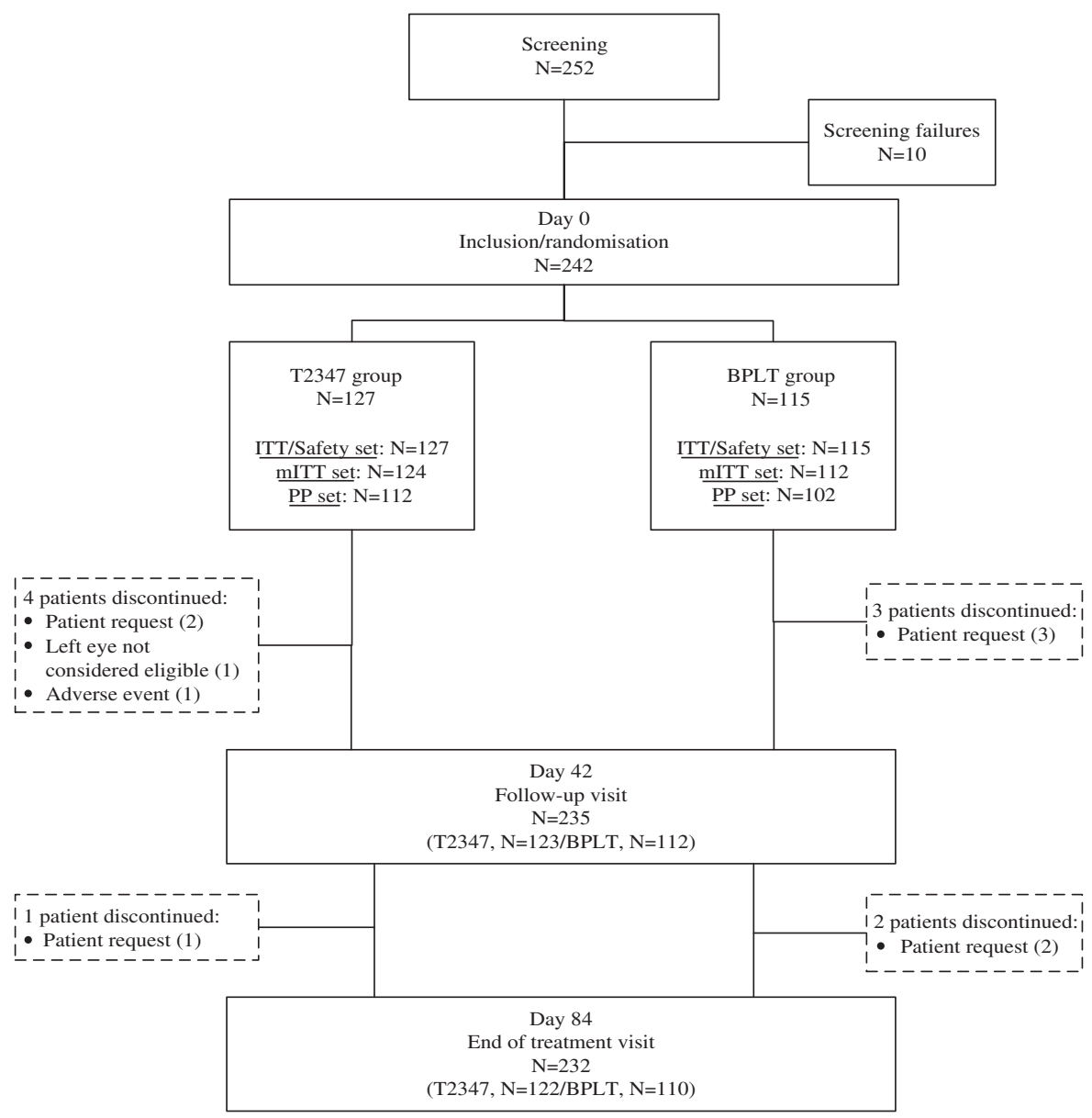

FIGURE 1. Patient disposition. 


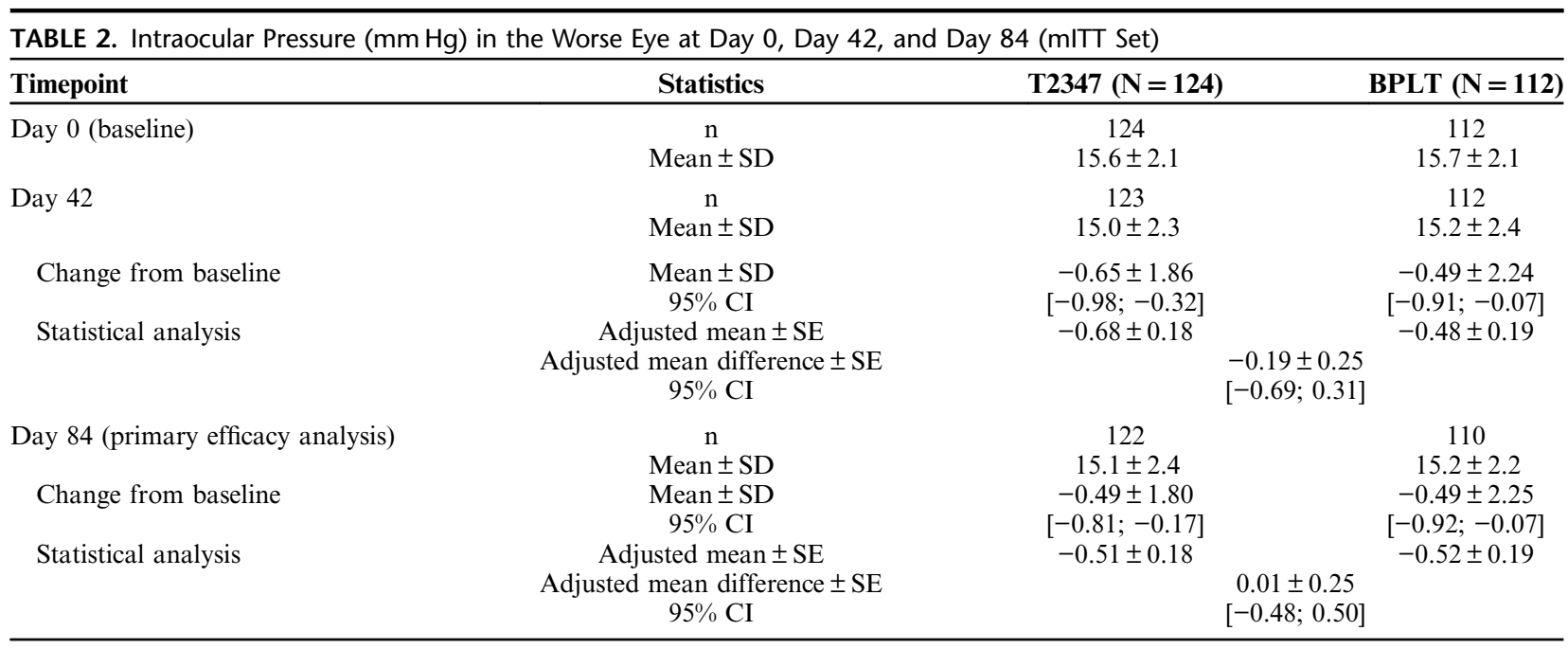

Adjusted mean difference $=($ T2347 minus BPLT $)$.

$\mathrm{CI}$ indicates confidence interval; N, number of patients in the group; n, number of patients with evaluable data; SE, standard error.

eye pain (one patient in the T2347 group) and eye irritation (one patient in each group).

\section{Ocular Symptoms}

The median (range) total severity score of ocular symptoms upon instillation was similar at baseline in the T2347 group and the BPLT group (1.0 [0-9] for both), and it was consistently lower for T2347 than for BPLT on D42 (0.0 [0-9] vs. 1.0 [0-6]) and D84 (0.0 [0-6] vs. 0.5 [0-7]). A statistically significant between-group difference was shown for the change from baseline in the total score of symptoms upon instillation on D42 $(P=0.007)$, as well as on D84 $(P=0.021)$ (Table 4$)$. No statistically significant betweengroup difference was shown for ocular symptoms throughout the day on D42 $(P=0.155)$ and D84 $(P=0.129)$ (Table 4).

There were no statistically significant differences between treatments in individual ocular symptoms of tearing, foreign body sensation, and eye dryness sensation on D42 or D84, either upon instillation or throughout the day (Fig. 2 and Table, Supplemental Digital Content 1, http://links.lww.com/ IJG/A245). However, irritation/burning/stinging was significantly less severe in the worse eye for T2347 than for BPLT upon instillation on D42 $(P=0.003)$ and D84 $(P<0.001)$ (Fig. 2A), but there was no difference between treatments throughout the day on D42 $(P=0.612)$ or D84 $(P=0.094)$ (Fig. 2B). Itching in the worse eye on D84 was significantly less severe for T2347 than for BPLT, both upon instillation $(P=0.01)$ (Fig. 2A) and throughout the day $(P<0.001)$ (Fig. 2B).

\section{Other Safety Parameters}

There were no clinically important differences between groups for any slit-lamp examination, including conjunctival hyperemia and global ocular staining (Table 4), nor for corneal thickness, fundoscopy, visual field examinations, bestcorrected far visual acuity, blood pressure, or heart rate during the study.

Satisfaction with regard to local tolerance assessed by the investigator and patient was similar for each group at both D42 ( $\geq 96.4 \%$ very satisfactory/satisfactory, $P=0.442$ and $P=0.110$, respectively) and at D84 ( $\geq 93.6 \%$ very satisfactory/satisfactory, $P=0.058$ and $P=0.089$ ). Of note, the percentage of patients who were very satisfied was higher in the T2347 group than in the BPLT group on both D42 $(57.0 \%$ vs. $45.0 \%)$ and D84 (65.3\% vs. $50.0 \%)$.

\section{DISCUSSION}

In this phase III randomized, investigator-masked, and multicenter study, noninferiority of efficacy for a preservative-free latanoprost-timolol fixed combination (T2347) compared with BPLT was demonstrated at D84 in patients suffering from OAG/ OHT who were previously receiving the preserved formulation. Although the overall safety profile was comparable between the 2 groups, T2347 induced improvements in some ocular symptoms.

Although an increased bioavailability of BAKpreserved drugs has been reported, ${ }^{26-28}$ a growing body of evidence indicates no difference between BAK-preserved and BAK-free formulations. Comparable penetration of both tafluprost formulations has been shown in the aqueous humor of rabbits. ${ }^{29}$ Furthermore, numerous clinical trials have assessed the impact on efficacy of the removal of BAK from latanoprost, ${ }^{25}$ bimatoprost, ${ }^{30}$ tafluprost ${ }^{31}$ timolol, ${ }^{32}$ or bimatoprost/timolol fixed combination ${ }^{33,34}$ formulations and concluded that the efficacy of the BAK-free formulation was noninferior (or equivalent) to that obtained with the BAK-preserved formulation. Recently, Bhagat and colleagues performed a randomized, parallel-group, activecontrolled study in patients with OAG or OHT to compare the IOP-lowering efficacy of a novel fixed-dose combination of latanoprost $0.005 \% /$ timolol $0.5 \%$, preserved with zinc chloride and boric acid, with latanoprost (BAK-preserved, Xalatan; Pfizer Inc., New York, NY) or timolol (BAKpreserved, Timoptic; Merck \& Co Inc., Whitehouse Station, NJ) administered as monotherapy or concomitantly. ${ }^{23}$ The IOP-lowering efficacy of this new BAK-free latanoprost/ timolol fixed combination was similar to BAK-preserved latanoprost plus timolol administered concomitantly, and better than preserved latanoprost or timolol administered alone. Our results are consistent with the literature and add to these previous findings in terms of IOP control. Of note, 
TABLE 3. Summary of AEs Related to Treatment (Safety Set)

\begin{tabular}{|c|c|c|c|c|}
\hline \multirow[b]{2}{*}{ Preferred Term } & \multicolumn{2}{|c|}{$\mathrm{T} 2347(\mathrm{~N}=127)$} & \multicolumn{2}{|c|}{$\operatorname{BPLT}(\mathrm{N}=115)$} \\
\hline & No. AEs & $\begin{array}{l}\text { Patients } \\
\text { [n (\%)] }\end{array}$ & No. AEs & $\begin{array}{l}\text { Patients } \\
\text { [n (\%)] }\end{array}$ \\
\hline Ocular AEs & 14 & $12(9.4)$ & 14 & $8(7.0)$ \\
\hline Eye irritation & 2 & $2(1.6)$ & 5 & $5(4.3)$ \\
\hline $\begin{array}{l}\text { Conjunctival } \\
\text { hyperemia }\end{array}$ & 3 & $3(2.4)$ & 1 & $1(0.9)$ \\
\hline Eye pain & 2 & $2(1.6)^{*}$ & 0 & 0 \\
\hline Eye allergy & 0 & 0 & 3 & $1(0.9)$ \\
\hline Eye discharge & 0 & 0 & 1 & $1(0.9)$ \\
\hline $\begin{array}{l}\text { Foreign body } \\
\text { sensation in eyes }\end{array}$ & 0 & 0 & 1 & $1(0.9)$ \\
\hline Keratitis & 0 & 0 & 1 & $1(0.9)$ \\
\hline Lacrimation increased & 0 & 0 & 1 & $1(0.9)$ \\
\hline $\begin{array}{l}\text { Intraocular pressure } \\
\text { increased }\end{array}$ & 0 & 0 & 1 & $1(0.9)$ \\
\hline Blepharitis & 1 & $1(0.8)$ & 0 & 0 \\
\hline Conjunctivitis staining & 1 & $1(0.8)$ & 0 & 0 \\
\hline Eye pruritus & 1 & $1(0.8)$ & 0 & 0 \\
\hline Ocular hyperaemia & 1 & $1(0.8)$ & 0 & 0 \\
\hline Optic disc hemorrhage & 1 & $1(0.8)^{*}$ & 0 & 0 \\
\hline $\begin{array}{l}\text { Papilloma } \\
\text { conjunctival }\end{array}$ & 1 & $1(0.8)^{*}$ & 0 & 0 \\
\hline Visual acuity reduced & 1 & $1(0.8)$ & 0 & 0 \\
\hline Systemic AEs & 4 & $4(3.1)$ & 2 & $2(1.7)$ \\
\hline Heart rate irregular & 0 & 0 & 1 & $1(0.9)$ \\
\hline Myalgia & 0 & 0 & 1 & $1(0.9)^{*}$ \\
\hline Arrhythmia & 1 & $1(0.8)$ & 0 & 0 \\
\hline Dysgeusia & 1 & $1(0.8)$ & 0 & 0 \\
\hline Fatigue & 1 & $1(0.8)$ & 0 & 0 \\
\hline Rhinorrhea & 1 & $1(0.8)^{*}$ & 0 & 0 \\
\hline
\end{tabular}

Preferred term coding according to the Medical Dictionary for Regulatory Activities Version 16.1 (September 2013).

Data are the number of events and number of patients (\%) with at least one treatment-related (unlikely, possible, or definite) episode.

*Relationship to treatment rated as unlikely (relationship of one of the two episodes of eye pain [T2347 group] rated as unlikely).

$\mathrm{N}$ indicates the number of patients in the group. the slight IOP reduction in patients who continued on the same treatment during the study (BPLT group) could be explained by the lack of reproducibility in diurnal IOP pattern over time $\mathrm{e}^{35,36}$ or by the Hawthorne effect, that is, better patient management and overall compliance in a clinical trial setting. ${ }^{37,38}$

Formulations without preservatives have previously been shown to have improved tolerability compared with those containing BAK. ${ }^{25}$ In the present study, however, preservative-free $\mathrm{T} 2347$ showed a similar overall safety profile to the BAK-preserved comparator. At least 4 possible explanations can be put forward for this observation. First, an 84-day treatment period may not be sufficient to observe real differences in objective ocular signs. For instance, in another 3-month study, a BAK-preserved formulation of travoprost showed a similar safety profile to a BAK-free formulation. ${ }^{39}$ In clinical practice, glaucoma treatment is usually given over a period of years, and the toxic effects of BAK may require regular use over a long period of time before adverse ocular signs are clinically identifiable. ${ }^{20,40}$ Second, although the combination of 2 ocular therapies might be expected to result in a safety profile reflecting the sum of side effects due to the individual components, clinical studies and meta-analyses have shown fixed combinations containing a $\beta$-blocker to be better tolerated than individual components. ${ }^{15,16,41,42}$ In this context, it is noteworthy that local tolerance, including moderate to severe conjunctival hyperemia, has been shown to be significantly improved by the same preservative-free formulation as T2347 but without timolol (Monoprost; Laboratoires Théa, Clermont-Ferrand, France) compared with BPLT without timolol (Xalatan) after 3 months of treatment. $^{25}$ The presence of timolol in the latanoprost formulations, therefore, could reduce the between-group difference in tolerability in the present study that would otherwise be expected due to the preservative. Third, patients with a more severe ocular surface disease were not included in the present study. Exclusion of these potentially more sensitive patients could have further limited the

TABLE 4. Summary of Main Ocular Safety Assessments (Safety set)

Change From Baseline (in 3 classes)

\begin{tabular}{|c|c|c|c|c|c|c|c|c|}
\hline & \multicolumn{4}{|c|}{ Total Symptom Score } & \multicolumn{4}{|c|}{ Ocular Signs } \\
\hline & \multicolumn{2}{|c|}{ Upon Instillation } & \multicolumn{2}{|c|}{ Throughout the Day } & \multicolumn{2}{|c|}{ Conjunctival Hyperaemia } & \multicolumn{2}{|c|}{ Global Ocular Staining } \\
\hline & $\mathbf{T} 2347$ & BPLT & $\mathbf{T} 2347$ & BPLT & $\mathbf{T} 2347$ & BPLT & T2347 & BPLT \\
\hline \multicolumn{9}{|l|}{ Day 42} \\
\hline $\mathrm{n}$ & 122 & 112 & 122 & 111 & 123 & 112 & 123 & 112 \\
\hline Improvement & $54(44.3)$ & $30(26.8)$ & $48(39.3)$ & $37(33.3)$ & $35(28.5)$ & $19(17.0)$ & $40(32.5)$ & $33(29.5)$ \\
\hline No change & $48(39.3)$ & $55(49.1)$ & 63 (51.6) & $60(54.1)$ & 77 (62.6) & $83(74.1)$ & $62(50.4)$ & $60(53.6)$ \\
\hline Worsening & $20(16.4)$ & $27(24.1)$ & $11(9.0)$ & 14 (12.6) & $11(8.9)$ & $10(8.9)$ & $21(17.1)$ & $19(17.0)$ \\
\hline$P$ & \multicolumn{2}{|c|}{0.007} & \multicolumn{2}{|c|}{0.155} & \multicolumn{2}{|c|}{0.135} & \multicolumn{2}{|c|}{0.535} \\
\hline \multicolumn{9}{|l|}{ Day 84} \\
\hline $\mathrm{n}$ & 122 & 110 & 122 & 110 & 123 & 112 & 122 & 110 \\
\hline Improvement & $56(45.9)$ & $37(33.6)$ & $50(41.0)$ & $42(38.2)$ & $39(32.0)$ & $23(20.9)$ & $42(34.4)$ & $42(38.2)$ \\
\hline No change & $54(44.3)$ & $52(47.3)$ & $65(53.3)$ & $51(46.4)$ & $73(59.8)$ & $75(68.2)$ & $62(50.8)$ & $52(47.3)$ \\
\hline Worsening & $12(9.8)$ & $21(19.1)$ & $7(3.0)$ & $17(15.5)$ & $10(8.2)$ & $12(10.9)$ & $18(14.8)$ & $16(14.5)$ \\
\hline$P$ & \multicolumn{2}{|c|}{0.021} & \multicolumn{2}{|c|}{0.129} & \multicolumn{2}{|c|}{0.065} & \multicolumn{2}{|c|}{0.883} \\
\hline
\end{tabular}

Data are number (\%) of patients.

Number of patients in group: $\mathrm{N}=127$ for $\mathrm{T} 2347$ group; $\mathrm{N}=115$ for BPLT group.

$\mathrm{n}$ indicates number of patients with available data. 

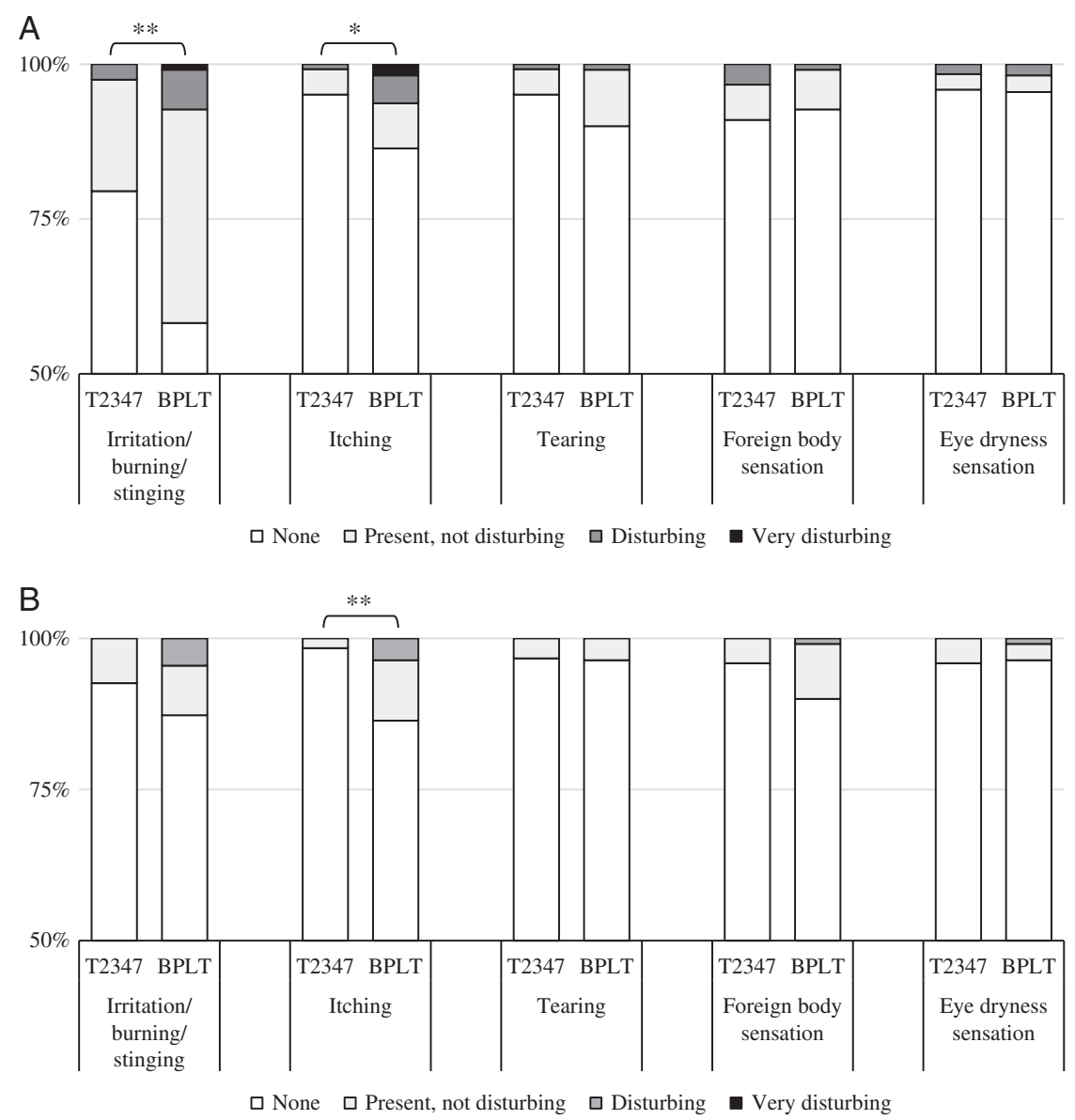

FIGURE 2. Individual ocular symptoms at day 84. Individual ocular symptom scores upon instillation (A) and throughout the day (B) were compared between groups using a Cochran Mantel Haenszel test with modified ridit scores stratified by country. ${ }^{*} P=0.01$; ${ }^{* \star} P<0.001$ (see also Supplement Table for details, Supplemental Digital Content 1, http://links.Iww.com/IJG/A245).

differences observed between groups over the 84-day study period. Finally, only patients already successfully treated with a preserved latanoprost-timolol fixed combination were included, and, by definition, these patients tolerated it well.

A potential limitation of the present study was the choice of a single morning IOP assessment for comparing T2347 to the preserved comparator. However, this approach is supported by several arguments: (1) peak IOP generally occurs in the morning in most POAG and OHT patients, (2) the 24-hour efficacy on IOP of the latanoprost-timolol fixed combination administered once daily in the evening in glaucoma and OHT patients is well established, (3) on the basis of preclinical data in monkeys, T2347 was shown as effective as BPLT in reducing IOP in the morning, as well as over 24 hours, after once-daily repeat dosing in the evening. ${ }^{43}$ In the preclinical study, the morning time point was shown to be the most challenging time to demonstrate noninferiority of T2347 to the reference treatment. This is consistent with the results from a cross-over clinical trial that showed a similar diurnal IOPlowering effect (measured at $8 \mathrm{AM}, 12$ noon, 4 PM, and 8 PM) of the same preservative-free formulation as T2347 but without timolol (Monoprost) in comparison with BPLT without timolol (Xalatan). ${ }^{44}$ Thus, it was considered that the IOP-lowering effect of T2347 compared with the reference BPLT could be relevantly extrapolated from the single morning IOP assessment.
Other potential limitations of the study included a doublemasked design not being feasible due to the different packaging of T2347 and BPLT (single-dose units vs. multidose vials, respectively). Although the study procedures ensured that single-masking was maintained and controlled by assigning personnel other than the investigator to dispense treatments and by instructing patients not to disclose treatment information to the investigator, patients could have known which treatment was assigned, which could have led to bias in the reporting of subjective assessments. Also, for ethical purposes, a washout period was deemed not to be appropriate for these patients already treated and IOP controlled by preserved fixed combination at study entry. Moreover, the primary endpoint was evaluated at D84, allowing sufficient time to eliminate any carry-over effect of previous treatment. With regard to the statistical tests, an upper $95 \%$ CI limit of $1.5 \mathrm{~mm} \mathrm{Hg}$ for the treatment difference at D84 was used to show noninferiority for the IOP change from baseline for T2347 compared with BPLT. While $1.5 \mathrm{~mm} \mathrm{Hg}$ is a common limit for studies with participants with uncontrolled IOP at baseline, ${ }^{15,45}$ using it for participants already medicated at study entry and therefore with lower IOP at baseline, such as in the present study, could be considered a less rigorous standard. However, the noninferiority of T2347 would still have been demonstrated using the more stringent limit of $1 \mathrm{~mm} \mathrm{Hg},{ }^{46,47}$ and even using $0.5 \mathrm{~mm} \mathrm{Hg}$. In addition, similar results were observed at D42, 
although the study was not designed for a formal noninferiority assessment at this time point.

In conclusion, the preservative-free latanoprost-timolol fixed combination T2347 showed noninferior efficacy compared with the preserved comparator in patients who were previously receiving the preserved formulation at study entry. T2347 was well tolerated and demonstrated improvements in some of the ocular symptoms. It could potentially confer a therapeutic advantage for $\mathrm{OAG} / \mathrm{OHT}$ patients on long-term treatment.

\section{ACKNOWLEDGMENTS}

The authors acknowledge the patients for their participation in this study and all study site personnel.

The authors thank Dr Andrew Lane (Lane Medical Writing) who provided professional medical writing assistance in the preparation and development of this manuscript in accordance with the European Medical Writers Association guidelines and Good Publication Practice.

The research contribution from Dr Clarke was supported by the NIHR Biomedical Research Centre at Moorfields Eye Hospital NHS Foundation Trust, UCL Institute of Ophthalmology, and the NIHR Moorfields Clinical Research Facility. The views expressed are those of the authors and not necessarily those of the NHS, the NIHR, or the Department of Health.

The authors acknowledge the T2347 Study Group, composed of: Belgium: Dr Nathalie Collignon, Dr Marc Goethals, Prof Ingeborg Stalmans; Estonia: Dr Tiia Jugaste, Dr Kuldar Kaljurand, Dr Krista Turman; France: Prof Béatrice Cochener, Dr Laurent Coupier, Prof Bruno Mortemousque, Prof JeanPhilippe Nordmann, Prof Jean-François Rouland, Dr MarieClaude Veschambre; Germany: Dr Philipp Franko Zeitz, Prof Norbert Pfeiffer, Dr Stefanie Schmickler, Dr Bogomil Voykov, Dr Joachim Watchlin; Hungary: Dr Adrienne Csutak, Dr Alexis Tsorbatzoglou, Prof Péter Vámosi, Dr L. Balázs Varsányi; Latvia: Dr Lasma Volksone; Poland: Prof Bartlomiej Kaluzny, Prof Ewa Mrukwa-Kominek, Dr Joanna Konopinska, Prof Wojciech Lubinski, Dr Jaroslaw Marek, Prof Jerzy Szaflik, Prof Tomasz Zarnowski; Russia: Prof Yury S. Astakhov, Prof Valeriy P. Erichev, Dr Andrey Zolotarev; Spain: Prof Alfonso Antón-López, Dr Elena Arrondo, Prof Javier Benitez-Del-Castillo, $\mathrm{Dr}$ Rafael A. Giménez-Gómez, Prof Noemi Güerri, Prof Enrique Mencía-Gutiérrez, $D r$ Jesús Hernández-Barahona Palma, Dr Jerónimo J. Lajara-Blesa, Dr Cosme Lavín-Dapena, Prof Javier Moreno-Montañés, Dr Ignacio Vinuesa-Silva; UK: Prof David C. Broadway, Dr Jonathan Clarke, Dr Mohammed Hassan, Dr James Kirwan, Dr Donald Montgomery.

\section{REFERENCES}

1. European Glaucoma Society. Terminology and guidelines for glaucoma, 4th Edition; 2014. Available at: https://www.eugs. org/eng/guidelines.asp. Accessed February 27, 2019.

2. Kass MA, Heuer DK, Higginbotham EJ, et al. The Ocular Hypertension Treatment Study: a randomized trial determines that topical ocular hypotensive medication delays or prevents the onset of primary open-angle glaucoma. Arch Ophthalmol. 2002;120:701-713; discussion 829-830.

3. Barnebey HS, Robin AL. Adherence to fixed-combination versus unfixed travoprost $0.004 \% /$ timolol $0.5 \%$ for glaucoma or ocular hypertension: a randomized trial. Am J Ophthalmol. 2017;176:61-69.

4. Konstas AG, Maskaleris G, Gratsonidis S, et al. Compliance and viewpoint of glaucoma patients in Greece. Eye (Lond). 2000;14(Pt 5):752-756.
5. Olthoff CM, Schouten JS, van de Borne BW, et al. Noncompliance with ocular hypotensive treatment in patients with glaucoma or ocular hypertension an evidence-based review. Ophthalmology. 2005;112:953-961.

6. Robin AL, Covert D. Does adjunctive glaucoma therapy affect adherence to the initial primary therapy? Ophthalmology. 2005;112:863-868.

7. Hollo G, Topouzis F, Fechtner RD. Fixed-combination intraocular pressure-lowering therapy for glaucoma and ocular hypertension: advantages in clinical practice. Expert Opin Pharmacother. 2014;15:1737-1747.

8. Hollo G, Katsanos A, Boboridis KG, et al. Preservative-free prostaglandin analogs and prostaglandin/timolol fixed combinations in the treatment of glaucoma: efficacy, safet, and potential advantages. Drugs. 2018;78:39-64.

9. Bron A, Baudouin C, Denis P, et al. Satisfaction and compliance of ocular hypertensive and glaucoma patients topically treated with a combination therapy. $J$ Fr Ophtalmol. 2008:31:659-665.

10. Higginbotham EJ. Considerations in glaucoma therapy: fixed combinations versus their component medications. Clin Ophthalmol. 2010;4:1-9.

11. Cheng JW, Cheng SW, Gao LD, et al. Intraocular pressurelowering effects of commonly used fixed-combination drugs with timolol: a systematic review and meta-analysis. PloS One. 2012;7:e45079.

12. Bangalore S, Kamalakkannan G, Parkar S, et al. Fixed-dose combinations improve medication compliance: a meta-analysis. Am J Med. 2007;120:713-719.

13. Inoue K, Okayama R, Higa R, et al. Ocular hypotensive effects and safety over 3 months of switching from an unfixed combination to latanoprost $0.005 \% /$ timolol maleate $0.5 \%$ fixed combination. J Ocul Pharmacol Therapeut. 2011;27: $581-587$.

14. Hamacher T, Schinzel M, Scholzel-Klatt A, et al. Short term efficacy and safety in glaucoma patients changed to the latanoprost $0.005 \% /$ timolol maleate $0.5 \%$ fixed combination from monotherapies and adjunctive therapies. Br J Ophthalmol. 2004;88:1295-1298.

15. Quaranta L, Biagioli E, Riva I, et al. Prostaglandin analogs and timolol-fixed versus unfixed combinations or monotherapy for open-angle glaucoma: a systematic review and meta-analysis. J Ocul Pharmacol Therapeut. 2013;29:382-389.

16. Aptel F, Cucherat M, Denis P. Efficacy and tolerability of prostaglandin-timolol fixed combinations: a meta-analysis of randomized clinical trials. Eur J Ophthalmol. 2012;22:5-18.

17. Sambhara D, Aref AA. Glaucoma management: relative value and place in therapy of available drug treatments. Ther $A d v$ Chronic Dis. 2014;5:30-43.

18. Horsley MB, Kahook MY. Effects of prostaglandin analog therapy on the ocular surface of glaucoma patients. Clin Ophthalmol. 2009;3:291-295.

19. Kastelan S, Tomic M, Metez Soldo K, et al. How ocular surface disease impacts the glaucoma treatment outcome. Biomed Res Int. 2013;2013:696328.

20. Baudouin C, Labbe A, Liang $\mathrm{H}$, et al. Preservatives in eyedrops: the good, the bad and the ugly. Progr Ret Eye Res. 2010;29:312-334.

21. Jaenen N, Baudouin C, Pouliquen P, et al. Ocular symptoms and signs with preserved and preservative-free glaucoma medications. Eur J Ophthalmol. 2007;17:341-349.

22. Baudouin C, Riancho L, Warnet JM, et al. In vitro studies of antiglaucomatous prostaglandin analogues: travoprost with and without benzalkonium chloride and preserved latanoprost. Investig Ophthalmol Vis Sci. 2007;48:4123-4128.

23. Bhagat P, Sodimalla K, Paul C, et al. Efficacy and safety of benzalkonium chloride-free fixed-dose combination of latanoprost and timolol in patients with open-angle glaucoma or ocular hypertension. Clin Ophthalmol. 2014;8:1241-1252.

24. European Medicines Agency. EMEA public statement on antimicrobial preservatives in ophthalmic preparations for human use. Doc. Ref.: EMEA/622721/2009. Available at: http://www.ema. 
europa.eu/docs/en_GB/document_library/Public_statement/2009/ 12/WC500017608.pdf. Accessed February 27, 2019.

25. Rouland JF, Traverso CE, Stalmans I, et al. Efficacy and safety of preservative-free latanoprost eyedrops, compared with BAKpreserved latanoprost in patients with ocular hypertension or glaucoma. Br J Ophthalmol. 2013;97:196-200.

26. Burstein NL. Preservative alteration of corneal permeability in humans and rabbits. Investig Ophthalmol Vis Sci. 1984;25: $1453-1457$.

27. Okabe K, Kimura H, Okabe J, et al. Effect of benzalkonium chloride on transscleral drug delivery. Investig Ophthalmol Vis Sci. 2005;46:703-708.

28. Majumdar S, Hippalgaonkar K, Repka MA. Effect of chitosan, benzalkonium chloride and ethylenediaminetetraacetic acid on permeation of acyclovir across isolated rabbit cornea. Int $J$ Pharm. 2008;348:175-178.

29. Pellinen P, Lokkila J. Corneal penetration into rabbit aqueous humor is comparable between preserved and preservative-free tafluprost. Ophthalmic Res. 2009;41:118-122.

30. Day DG, Walters TR, Schwartz GF, et al. Bimatoprost $0.03 \%$ preservative-free ophthalmic solution versus bimatoprost $0.03 \%$ ophthalmic solution (Lumigan) for glaucoma or ocular hypertension: a 12-week, randomised, double-masked trial. $\mathrm{Br} J$ Ophthalmol. 2013;97:989-993.

31. Hamacher T, Airaksinen J, Saarela V, et al. Efficacy and safety levels of preserved and preservative-free tafluprost are equivalent in patients with glaucoma or ocular hypertension: results from a pharmacodynamics analysis. Acta Ophthalmol Suppl (Oxf). 2008;242:14-19.

32. Easty DL, Nemeth-Wasmer G, Vounatsos JP, et al. Comparison of a non-preserved $0.1 \% \mathrm{~T}-\mathrm{Gel}$ eye gel (single dose unit) with a preserved $0.1 \% \mathrm{~T}$-Gel eye gel (multidose) in ocular hypertension and glaucomatous patients. $\mathrm{Br} J$ Ophthalmol. 2006;90:574-578.

33. Cordeiro MF, Goldberg I, Schiffman R, et al. Efficacy of a preservative-free formulation of fixed-combination bimatoprost and timolol (Ganfort PF) in treatment-naive patients vs previously treated patients. Clin Ophthalmol. 2015;9:1605-1611.

34. Goldberg I, Gil Pina R, Lanzagorta-Aresti A, et al. Bimatoprost $0.03 \% /$ timolol $0.5 \%$ preservative-free ophthalmic solution versus bimatoprost $0.03 \% /$ timolol $0.5 \%$ ophthalmic solution (Ganfort) for glaucoma or ocular hypertension: a 12-week randomised controlled trial. Br J Ophthalmol. 2014;98:926-931.

35. Realini T, Weinreb RN, Wisniewski S. Short-term repeatability of diurnal intraocular pressure patterns in glaucomatous individuals. Ophthalmology. 2011;118:47-51.
36. Aptel F, Lesoin A, Chiquet C, et al. Long-term reproducibility of diurnal intraocular pressure patterns in patients with glaucoma. Ophthalmology. 2014;121:1998-2003.

37. Delval L, Baudouin C, Gabisson P, et al. Safety and efficacy of unpreserved timolol $0.1 \%$ gel in patients controlled by preserved latanoprost with signs of ocular intolerance. Journal Francais d'Ophtalmologie. 2013;36:316-323.

38. Olander K, Zimmerman TJ, Downes N, et al. Switching from latanoprost to fixed-combination latanoprost-timolol: a 21-day, randomized, double-masked, active-control study in patients with glaucoma and ocular hypertension. Clin Ther. 2004;26: 1619-1629.

39. Lewis RA, Katz GJ, Weiss MJ, et al. Travoprost $0.004 \%$ with and without benzalkonium chloride: a comparison of safety and efficacy. J Glaucoma. 2007;16:98-103.

40. Aihara M, Otani S, Kozaki J, et al. Long-term effect of BAKfree travoprost on ocular surface and intraocular pressure in glaucoma patients after transition from latanoprost. J Glaucoma. 2012;21:60-64.

41. Radcliffe NM. The impact of timolol maleate on the ocular tolerability of fixed-combination glaucoma therapies. Clin Ophthalmol. 2014;8:2541-2549.

42. Vinuesa-Silva JM, Vinuesa-Silva I, Pinazo-Duran MD, et al [Development of conjunctival hyperemia with the use of a fixed combination of latanoprost/timolol: systematic review and meta-analysis of clinical trials]. Arch Soc Esp Oftalmol. 2009;84: 199-207.

43. Goody RJ, Woodley V, Henry S, et al. Intraocular pressure lowering effects of T2347 in normotensive nonhuman primates following once-daily topical administration. Poster presented at ARVO Annual Meeting, April 29-May 3, 2018, Honolulu, Hawaii.

44. Aptel F, Choudhry R, Stalmans I. Preservative-free versus preserved latanoprost eye drops in patients with open-angle glaucoma or ocular hypertension. Curr Med Res Opin. 2016; 32:1457-1463.

45. Strohmaier K, Snyder E, DuBiner H, et al. The efficacy and safety of the dorzolamide-timolol combination versus the concomitant administration of its components. DorzolamideTimolol Study Group. Ophthalmology. 1998;105:1936-1944.

46. Chauhan BC, Mikelberg FS, Balaszi AG, et al. Canadian Glaucoma Study: 2 risk factors for the progression of openangle glaucoma. Arch Ophthalmol. 2008;126:1030-1036.

47. Leske MC, Heijl A, Hussein M, et al. Factors for glaucoma progression and the effect of treatment: the early manifest glaucoma trial. Arch Ophthalmol. 2003;121:48-56. 